\title{
Introduction to the Special Issue on ASSETS'18
}

We are pleased to present three articles that are extended versions of conference papers presented at the 20th International ACM SIGACCESS Conference on Computers and Accessibility (ASSETS'18), which was held in Galway, Ireland, October 22-24, 2018. Authors of several top papers from the conference submitted manuscripts for consideration, which underwent a full review process for ACM's Transactions on Accessible Computing (TACCESS). The guest editor for this issue is Karyn Moffatt (McGill University, Montréal, Canada). In addition, Shari Trewin (IBM Research, USA) helped vet initial proposals from authors, providing valuable insight and feedback.

This issue includes the first three of these articles that have been accepted to this special issue of TACCESS. Additional articles based on ASSETS'18 papers may appear in a future issue of TACCESS. The journal editors thank the authors for their excellent submissions, and we also thank all of the journal reviewers who contributed their time and expertise to this process.

The first article, "Design for Social Accessibility Method Cards: Engaging Users and Reflecting on Social Scenarios for Accessible Design," describes and evaluates the Design for Social Accessibility perspective and an associated set of method cards. Results from a series of four studies establish the effectiveness of the Design for Social Accessibility methods for helping students and designers emphasize social factors during the design process.

The second article, "Blind Leading Sighted: Drawing Design Insights from Blind Users Toward More Productivity-Oriented Voice Interfaces," presents the results of two studies that describe how blind users engage with voice activity personal assistants and detail the usability and accessibility issues encountered when using these devices. This work provides practical guidance on how to improve the design of voice activity personal assistants for both blind and sighted users.

The third article, "Deep Learning Compensation of Rotation Errors During Navigation Assistance for People with Visual Impairments or Blindness," establishes the impact and prevalence of rotation errors during navigation assistance and introduces a novel technique for reducing such errors. Evaluations with blind individuals establishes the effectiveness of this approach in reducing rotation errors.

Karyn Moffatt McGill University

Guest Editor

(c) 2019 Copyright held by the owner/author(s).

1936-7228/2019/12-ART17e

https://doi.org/10.1145/3372925 